\section{Nuclear Methods in Condensed Matter Studies}

\author{
K. Bethge, Frankfurt/Main \\ (Johann Wolfgang Goethe-Universität)
}

\begin{abstract}
The specific features of nuclear reactions can be used to determine quantity and distribution of elements in solid matter.
\end{abstract}

Nuclear reactions have been studied over decades to determine the properties of all available nuclei. This enormous amount of information can now be put to use to detect, i.e. locate and identify, nuclei in their natural or artificial surroundings in different host materials. The specific properties of nuclei which can be exploited are their de-excitation radiation, the emission of particles, or special scattering features like Coulomb or resonance scattering. The behaviour of nuclei corresponding to these properties is, in general, independent of their atomic and molecular state such as being incorporated in lattices.

For all nuclear methods of analysis it is important to know:

i) the reaction cross-section in the relevant energy range,

ii) the energy loss of charged particles before and after the reactions in the materials under study,

iii) the kinematics of the process.

From extensive studies in the past, the cross-sections are known and the energy loss has been measured for very many projectile/material combinations. Moreover, as the dominating electronic energy loss from interactions with atomic shell systems involves averaging over many electronic energy states, extrapolations can be made with a high degree of accuracy. The kinematics finally is based on the values of nuclear masses which are well established.

From a knowledge of these quantities it is possible to determine a profile, i.e. a distribution of atoms of a special element incorporated in a host matrix using the procedure illustrated in Fig. 1. A monoenergetic ion beam of energy $E_{0}$ is directed onto the surface of a matrix containing an element whose cross-section $\sigma$ is of the form shown upper left, i.e. with a resonance at $E_{R}$. As the incident particles penetrate the material they lose energy, but as $E_{\mathrm{R}}$ is approached, the absorption suddenly increases. Consequently a simple relation between counting rate and depth can be established. The narrower the resonance, the better is the spatial resolution of a profile.
Almost all possible reactions have been exploited in such studies ") but only a few examples will be mentioned of those which have already become established techniques ${ }^{2}$ ).

\section{Scattering}

Scattering processes are characterised by the interaction potential, that based on Coulomb charge interaction giving rise to the well known Rutherford scattering law. Rutherford back-scattering with $\alpha$-particles was first applied to materials studies by Turkevich et al. ${ }^{3}$ ) who determined the composition of the moon surface in unmanned space research as a prelude to the subsequent landings. Rutherford scattering in the backward mode is preferred because it gives the best charge resolution ${ }^{5}$ ). Particles coming from different depths have different energies and this produces a continuous spectrum. The conversion of the signals from scattering events into a depth profile has thus to take account of the energy loss. Fig. 2 shows such a spectrum for a silicon sample overlayed by titanum and iron deposited by sputtering, during which some oxygen became incorporated. The spectrum shows the unresolved $\mathrm{Fe}-\mathrm{Ti}$ layer as a
Fig. 1 - Schematic diagram of conversion of signals from scattering events into a depth profile. discrete peak at the highest $\alpha$-particle energies. Since $\mathrm{Fe}$ and $\mathrm{Ti}$ are much heavier than $\mathrm{He}$, the energy transfer from the bombarding $\alpha$-particles is smallest, and the $\alpha$-particles are scattered with little energy loss. Since the metal layer is thin, this gives rise to a single peak spectrum. The bulk material silicon, however, is thicker than the particle range and a continuous spectrum results with a cut-off at an energy corresponding to the smaller mass of silicon compared to $\mathrm{Ti}$ and $\mathrm{Fe}$.

Oxygen in our example is the lightest element and so gives rise to the largest energy transfer and a scattering spectrum at a lower energy still. The thickness and the depth of the oxygen atoms can be determined by such a scattering experiment and in the case described, a homogeneous oxygen content in the FeTi layer can be inferred from the spectrum breadth.

A special feature of the scattering is the sharp peak at the edge of the oxygen spectrum. This originates from the nuclear resonance scattering of $\alpha$-particles from oxygen at $3.045 \mathrm{MeV}$. As soon as the resonance energy has been passed normal scattering takes over. Such resonant scattering has been exploited particularly for the detection of oxygen and carbon.

Rutherford back-scattering is nowadays standard procedure in many solid state research projects. It entails an interesting combination of accelerators and detection methods with materials science research.

\section{Hydrogen Profiling}

Hydrogen is a very important impurity in many materials. Its presence causes important modifications to properties,

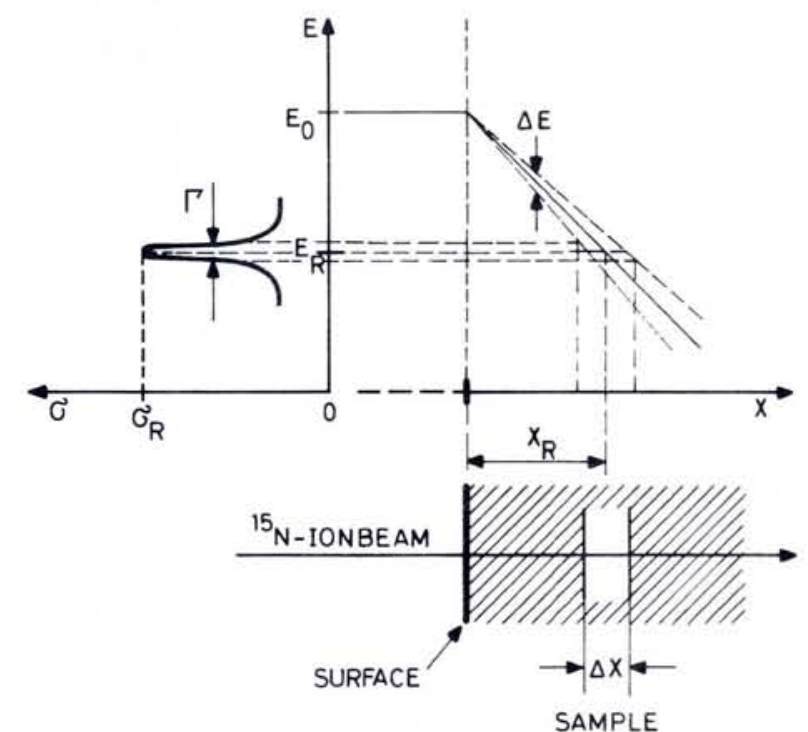




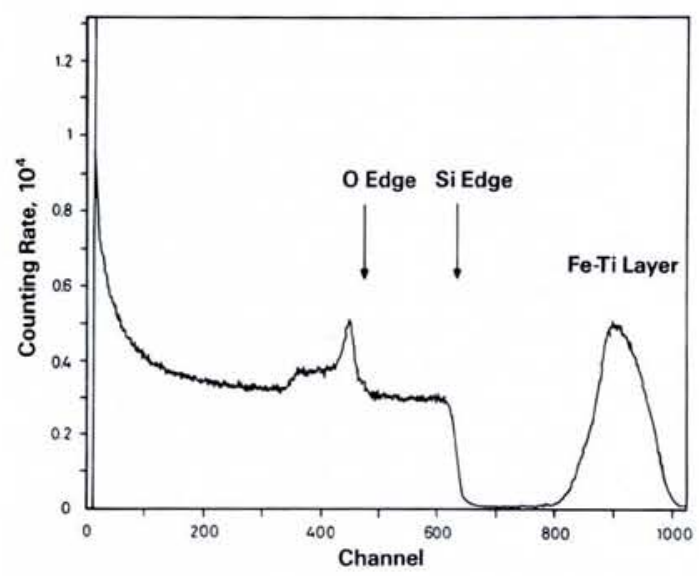

Fig. 2 - Analysis of a Si sample covered with a Fe-Ti layer.

for example in solar cells where non-stochiometric concentrations of hydrogen in silicon lead to improvements in the efficiency of energy conversion. Also foils of carbon-hydrogen combinations can have astonishing features, e.g. extreme hardness and long life under particle bombardment. In contrast, hydrogen corrosion is an unwanted but important feature in many metals. The determination of hydrogen is however difficult in regard to both concentration and profile. Radiation emitted by the atoms cannot be used because of its low energy and the capture cross-section for neutrons is very small. There are, however, nuclear reactions between protons and "fairly" light elements which can be used, some of which show narrow resonances in the excitation function, as e.g. ${ }^{1} \mathrm{H}\left({ }^{15} \mathrm{~N}, \alpha \gamma\right)$ ${ }^{12} \mathrm{C}$. This reaction shows a sharp resonance at $6.38 \mathrm{MeV}$.

The detection of $\gamma$-radiation emitted from the impact of a nitrogen-15 nucleus with a proton allows the hydrogen pro- file to be measured, following the procedure discussed above. A profile of hydrogen in a TiNi alloy covered by an $18 \mathrm{~nm}$ and $115 \mathrm{~nm}$ Pd layer is shown in Fig. 3. Hydrogen is only present in the TiNi alloy $\left.{ }^{6}\right)$. Since it is only necessary to detect $4.43 \mathrm{MeV} \gamma$-radiation from carbon-12, the whole experimental equipment can be kept simple. The detection limit for such analyses is the background radiation which is of the order of 0.1 at. $\%$ unless very special shielding precautions are taken.

\section{Nuclear Reaction Analysis}

The analysis of reaction products emitted as a consequence of bombardment can be used to determine impurities in implanted materials very selectively provided the relevant energy losses of the primary particles and emitted reaction products are known. The depth profile can then be obtained from an energy analysis of the ejected particles. Many different reactions have al- ready been employed and more still are presented at each of the many conferences devoted to the topic ${ }^{4}$ ).

One interesting application of nuclear reaction analysis that should be mentioned is the measurement of sputter yields of silicon under argon ion bombardment ${ }^{7}$ ). The experiment was performed in four steps:

1. Into a sample of silicon, nitrogen was implanted as a marker - an energy of $900 \mathrm{keV}$ corresponds to a depth of 1.8 $\mu \mathrm{m}$.

2. The profile of the implanted nitrogen layer was determined by analysis of the reaction ${ }^{14} \mathrm{~N}(\mathrm{~d}, \alpha)^{12} \mathrm{C}$ as illustrated in Fig. 4 where the first three excited levels of ${ }^{12} \mathrm{C}$ measured at $160^{\circ}$ are shown as solid lines.

3. The surface of the silicon sample was then sputtered off by argon ion bombardment at $15 \mathrm{keV}$ for several hours.

4. Finally the profile of the implanted nitrogen was determined again using the reaction ${ }^{14} \mathrm{~N}(\mathrm{~d}, \alpha){ }^{12} \mathrm{C}$. This resulted in the spectrum indicated by the dashed lines in Fig. 4.

From the energy shift of the lines and a knowledge of the energy loss of deuterons and $\alpha$-particles in silicon, the amount of material removed by sputtering could be determined with high accuracy. Without going into the details, this one example may show already the potential of nuclear reaction analysis in different fields of physics.

\section{Charged Particles Activation Analysis}

Information on the abundance of light elements like carbon, nitrogen and oxy-

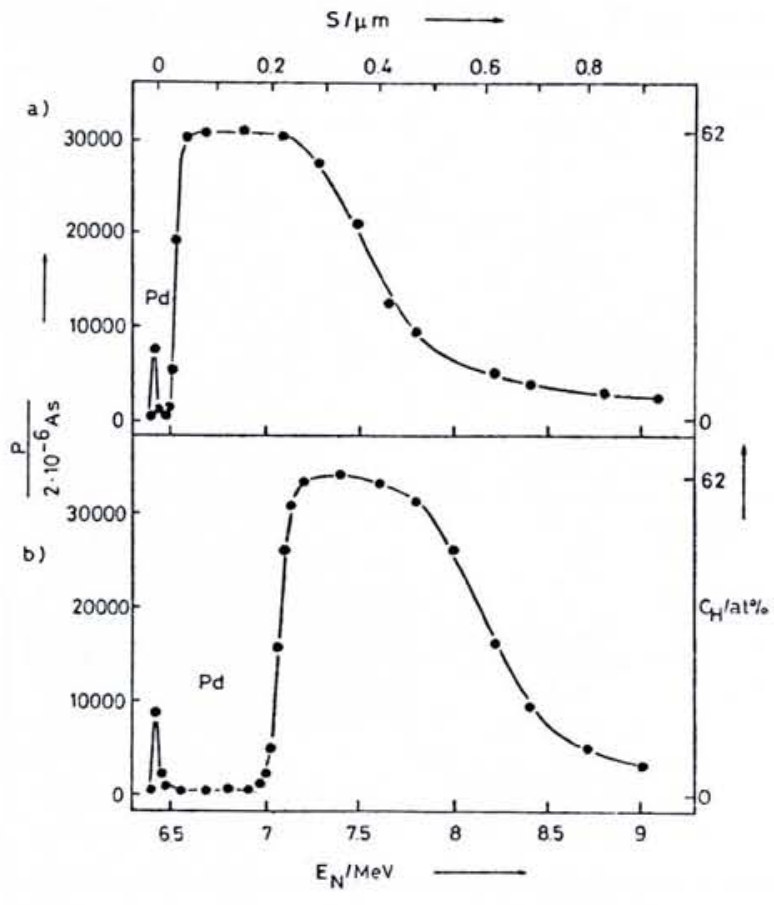

Fig. 3- Hydrogen profile of a TiNi alloy with Pd surface layer.

Fig. 4 - Spectra of ${ }^{14} \mathrm{~N}(d, \alpha){ }^{12} \mathrm{C}$ reaction: solid line before sputtering, dashed lined after sputtering.

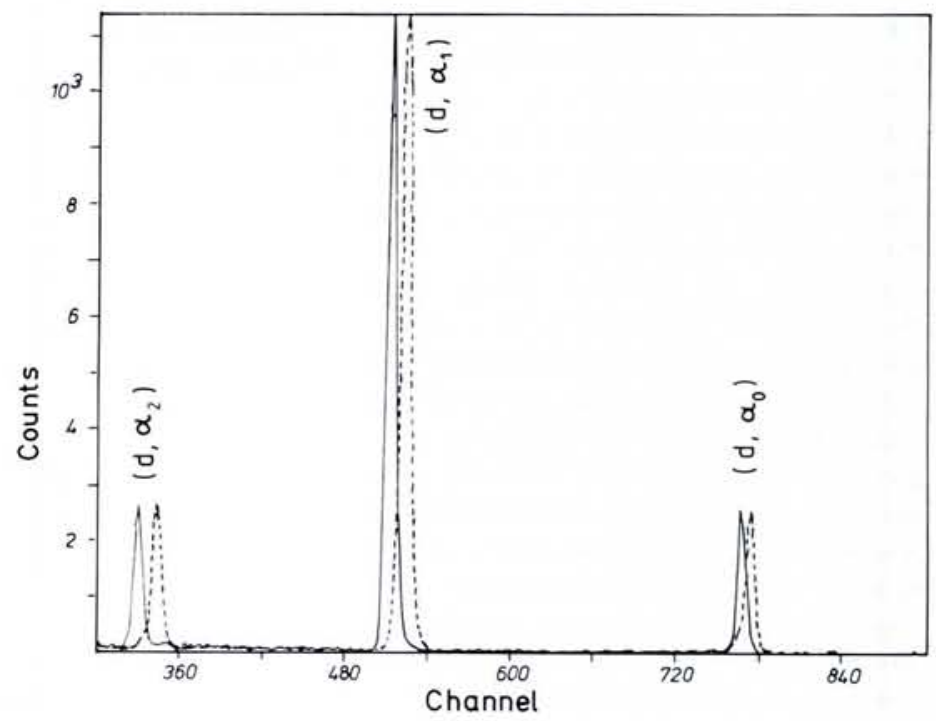




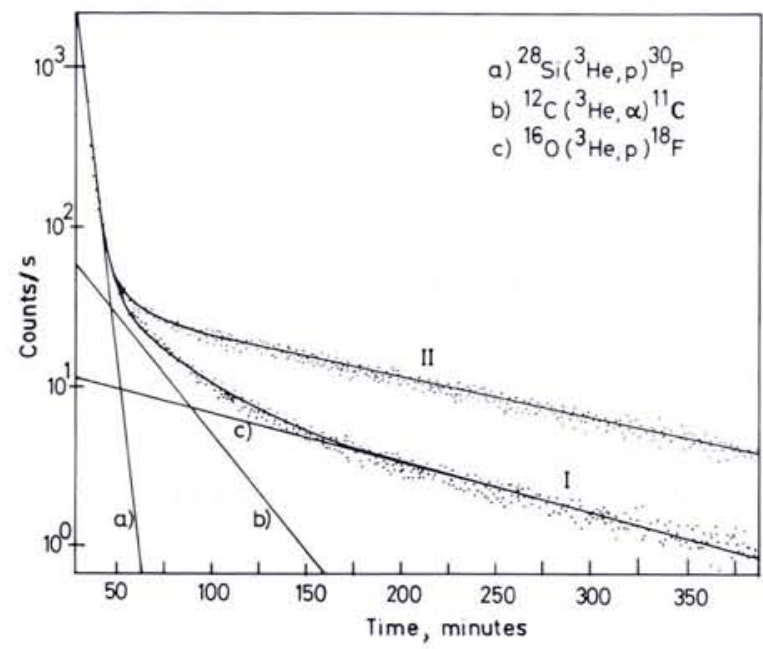

Fig. 5-Analysis of $C$ and $O$ in silicon by charged particle activation analysis.

gen in metals and semiconductors is essential for understanding the physical properties of these materials. The profiling of elements has already been mentioned, but it is worth considering the analysis method which yields results faster, even if only the integral number of impurity atoms is measured. All three elements mentioned above possess radioactive isotopes which decay by positron emission with reasonably short lifetimes, although long enough to guarantee statistically significant measurements. The reactions preferred are induced by ${ }^{3} \mathrm{He}$ particles as e.g. :

$$
\begin{array}{ll}
{ }^{12} \mathrm{C}\left({ }^{3} \mathrm{He}, \alpha\right)^{11} \mathrm{C} & \mathrm{t}_{1 / 2}=20.4 \mathrm{~min} \\
{ }^{14} \mathrm{~N}\left({ }^{3} \mathrm{He}, \alpha\right)^{13} \mathrm{~N} & \mathrm{t}_{1 / 2}=9.96 \mathrm{~min} \\
{ }^{16} \mathrm{O}\left({ }^{3} \mathrm{He}, \mathrm{p}\right)^{18} \mathrm{~F} & \mathrm{t}_{1 / 2}=109.7 \mathrm{~min}
\end{array}
$$

Activation times of the same order of magnitude as the decay lifetimes are required which means that this type of analysis is applicable to industrial conditions. Samples are activated by bombarding with ${ }^{3} \mathrm{He}$ particles of several $\mathrm{MeV}$ energy. Since the cross-section depends only slightly on energy in the range accessible to small accelerators, the bombarding energy is selected according to the depth from which information is wanted. In Fig. 5 the decay curves for two activated silicon samples are shown. I denotes a zone-refined silicon rod, whereas II denotes a Czochralskigrown silicon crystal. The decay curve a) indicates activation of the silicon matrix which decays with a half life of $2.5 \mathrm{~min}$. Curve b) gives the amount of carbon present in each sample, whereas c) allows the abundance of oxygen to be determined. The two samples produced by different processes contain oxygen concentrations which differ by an order of magnitude. As already mentioned, the time of analysis is reasonably short and the detection limits so far achieved are 8 $\mathrm{ppb}$ for carbon and $2 \mathrm{ppb}$ for oxygen in silicon. These values are far superior to those obtained with neutron activation.

\section{Final Remark}

The study of nuclear reactions over several decades has amassed a great deal of data about all existing isotopes. Many of them play an important role in materials science and in the solution of technical problems. Nuclear physics with its vast reservoir of information can contribute considerably to technical promark and companied the award read as follows: ballistic injection of electrons. gress, and the application of nuclear analysis methods is still growing. The improvement of detection limits is still an important task. Laboratories equipped with instrumentation for radiation detection and particle acceleration are well suited to carry out such investigations and many laboratories throughout the world are engaged in such work.

\section{REFERENCES}

1. Mayer J.W. and Rimini E., Ion Beam Handbook for Materials Anaysis, (Acad. Press, N.Y.) 1977.

2. Bethge K., Phys. BI. 40 (1984) 83.

3. Turkevich A.L., Franzgrote E.J. and Patterson J.H., Science 158 (1967) 636.

4. Proceedings of the Europhysics Conference on Nuclear Physics Methods in Materials Research, (Eds. K. Bethge, H. Baumann, H. Jex and F. Rauch) Darmstadt, Sept. 1980. 5. Chu W.K., Mayer J.W. and Nicolet M.A., Backscattering Spectrometry (Acad. Press. N.Y.) 1978.

6. Brauer E., Grüner R. and Rauch F., Ber. Bunsenges. Phys. Chem. 87 (1983) 341.

7. Weniger R., Dissertation Frankfurt (1984).

\section{EPS 1985 Hewlett-Packard Europhysics Prize}

As briefly announced in the January issue of Europhysics News the 1985 Hewlett-Packard Europhysics Prize has been awarded jointly to:

Dr. Jens Als-Nielsen of the Ris $\varnothing$ National Laboratory, Roskilde, Den-

Dr. Michael Pepper of the Cavendish Laboratory, Cambridge and the GEC's Hirst Research Centre, Wembley, UK.

The awards were presented on 20 March 1985 during the 5th EPS CMD General Conference held in Berlin (West). The official citation which ac-

"The importance of the dimensionality of physical systems has gained increased recognition in the last years both in condensed matter physics and material science, and in statistical physics. The work of the prize winners has in a significant way contributed to this. In particular they have shown that low-dimensional systems provide opportunities for ingenious experimentation to isolate and characterize physical features and new phenomena. The systems they have chosen for their studies have consisted of surfaces and suspended films of liquids, and of deposited layers and narrowly formed channels in metals and semiconductors. In studying these systems they have, often in a pioneering manner, applied refined techniques such as synchrotron $X$-ray diffraction and

From their research has come a wealth of information on novel types of collective states and quantum levels, such as the Peierls state and the quantized Hall effect. The results on the dependence of ordering and fluctuations on the dimensionality have in a gratifying manner verified recent developments in the theory of critical phenomena. The studies of electronic localization and transport in restricted or low-dimensional geometries have not only increased our understanding of these phenomena but may also lead to later technological applications.

The scientific achievements of Drs Als-Nielsen and Pepper within lowdimensional physics make them worthy recipients of the HewlettPackard Prize in condensed-matter physics for 1985."

Articles by the prize winners as well as a report on the CMD Conference will be carried in future issues of Europhysics News. 